\title{
Fluoroscopy with MRA fusion image guidance in endovascular iliac artery interventions: study protocol for a randomized controlled trial (3DMR-Iliac- roadmapping study)
}

Seline R. Goudeketting ${ }^{1 *}$ (D), Stefan G. H. Heinen ${ }^{1}$, Michiel W. de Haan², Anna M. Sailer ${ }^{3}$ Daniel A. F. van den Heuvel ${ }^{4}$, Marco J. van Strijen ${ }^{4}$ and Jean-Paul P. M. de Vries ${ }^{5}$

\begin{abstract}
Background: Endovascular iliac artery interventions rely on the use of two-dimensional digital subtraction angiographies with an iodinated contrast agent and ionizing radiation. The amount of iodinated contrast agent should be limited because of its potentially nephrotoxic effects. Three-dimensional (3D) image fusion requires registration of a preprocedural magnetic resonance angiogram (MRA) or computed tomography (CT) angiogram to a perprocedurally acquired cone-beam CT or two fluoroscopic orthogonal projections. After registration, the 3D angiography images can be overlaid on the fluoroscopy screen and will follow table and C-arm movements. This study will assess the added value of the 3D image fusion technique in iliac artery interventions regarding the amount of the iodinated contrast agent administered.

Methods/Design: The study cohort will comprise 106 patients (> 18 years) with symptomatic common and/or external iliac artery stenoses or occlusions and a recent ( $<6$ months) diagnostic MRA from the pelvis through the lower extremities, for which an endovascular intervention is indicated. Patients will be randomized into the control or study group (i.e. treatment without or with 3D image fusion guidance). The primary endpoint is the amount of administered iodinated contrast agent $(\mathrm{mL})$. Secondary outcomes are technical success of the procedure, defined as $<30 \%$ residual stenosis over the treated lesion, fluoroscopy time, and radiation dose as dose area product $\left(\mathrm{mGy} \mathrm{cm}^{2}\right)$. Patient participation in the study will be completed after hospital discharge.
\end{abstract}

Discussion: This study is a randomized controlled multicenter trial to provide evidence on the effect of the 3D image fusion technique on the amount of administered iodinated contrast during endovascular common and/or external iliac artery interventions.

Trial registration: Nederlands Trial Register, NTR5008. Registered on 16 December 2014.

Keywords: 3D imaging, Fusion imaging, Cone-beam computed tomography, Magnetic resonance angiography, lliac artery, Angioplasty

\footnotetext{
*Correspondence: s.goudeketting@antoniusziekenhuis.nl

'Department of Vascular Surgery, St. Antonius Hospital, Koekoekslaan 1, 3435

CM Nieuwegein, The Netherlands

Full list of author information is available at the end of the article
}

(c) The Author(s). 2018 Open Access This article is distributed under the terms of the Creative Commons Attribution 4.0 International License (http://creativecommons.org/licenses/by/4.0/), which permits unrestricted use, distribution, and reproduction in any medium, provided you give appropriate credit to the original author(s) and the source, provide a link to the Creative Commons license, and indicate if changes were made. The Creative Commons Public Domain Dedication waiver (http://creativecommons.org/publicdomain/zero/1.0/) applies to the data made available in this article, unless otherwise stated. 


\section{Background}

The iliac arteries are involved in up to $30 \%$ of patients with peripheral artery disease (PAD) [1]. The preferred treatment for most patients is endovascular revascularization (i.e. percutaneous transluminal angioplasty [PTA] or recanalization) with optional stent placement. Two-dimensional (2D) digital subtraction angiography (DSA) with iodinated contrast agent and ionizing radiation is the gold standard to visualize the location and severity of the lesion [1]. Because of its potentially nephrotoxic effects, especially in patients with pre-existing renal failure or diabetes mellitus, the amount of iodinated contrast agent should be limited $[2,3]$.

Non-invasive imaging acquired before the procedure, such as contrast-enhanced magnetic resonance angiography (MRA) or computed tomography angiography (CTA), can be used as guidance during these endovascular interventions. The three-dimensional (3D) image fusion technique is based on the rigid registration of MRA or CTA to a perprocedurally acquired cone-beam computed tomography (CBCT) or, alternatively, two fluoroscopic orthogonal images. This registration is based on bony landmarks, after which the MRA images will be visualized on the 2D fluoroscopy screen (Fig. 1). Because of the registration, the MRA images will follow table and $\mathrm{C}$-arm movements. The 3D image fusion technique does not require the use of additional iodinated contrast agent and has shown to significantly reduce the amount of iodinated contrast agent during endovascular abdominal aortic aneurysm repair (EVAR) [4-8].

No randomized controlled trials have been performed to assess the added value of the 3D image fusion technique in iliac artery interventions so far.

\section{Hypothesis}

The hypothesis of this trial is that the use of the 3D image fusion technique will result in a significant reduction of the amount of administered iodinated contrast agent during endovascular iliac artery interventions compared with the endovascular iliac artery procedures without the use of the 3D image fusion technique.

\section{Methods/Design \\ Study design}

This is a prospective randomized, controlled, multicenter trial. The flow diagram of the trial is shown in Fig. 2. The SPIRIT figure can be found in Fig. 3 and the checklist is available in Additional file 1.

\section{Study objectives and endpoints}

The primary endpoint is the amount of administered iodinated contrast agent $(\mathrm{mL})$. The intra-arterial iodinated contrast agents Ultravist $(300 \mathrm{mg} / \mathrm{mL}$; Bayer Vital, Leverkusen, Germany) and Xenetix $(300 \mathrm{mg} / \mathrm{mL}$; Guerbet, Roissy, France) will be used during all interventions of the Maastricht University Medical Center (MUMC) and St. Antonius Hospital (SAH), respectively.

Secondary endpoints are the following:

1. Technical success of the procedure, defined as < $30 \%$ trans-lesion residual stenosis and no flowlimiting dissections;

2. Fluoroscopy time from the start to end of the procedure in minutes and seconds;

3. Overall procedure time, defined as the time from femoral access to arterial closure (minutes);

4. Radiation dose as dose area product (DAP) in $\mathrm{mGycm}{ }^{2}$ from the start to the end of the procedure;

5. Fusion accuracy between the overlaid MRA and DSA, categorized as accurate $(<2 \mathrm{~mm})$, mismatch (2-5 mm), or inaccurate $(>5 \mathrm{~mm})$;

6. Complications that occur during the intervention and before discharge.

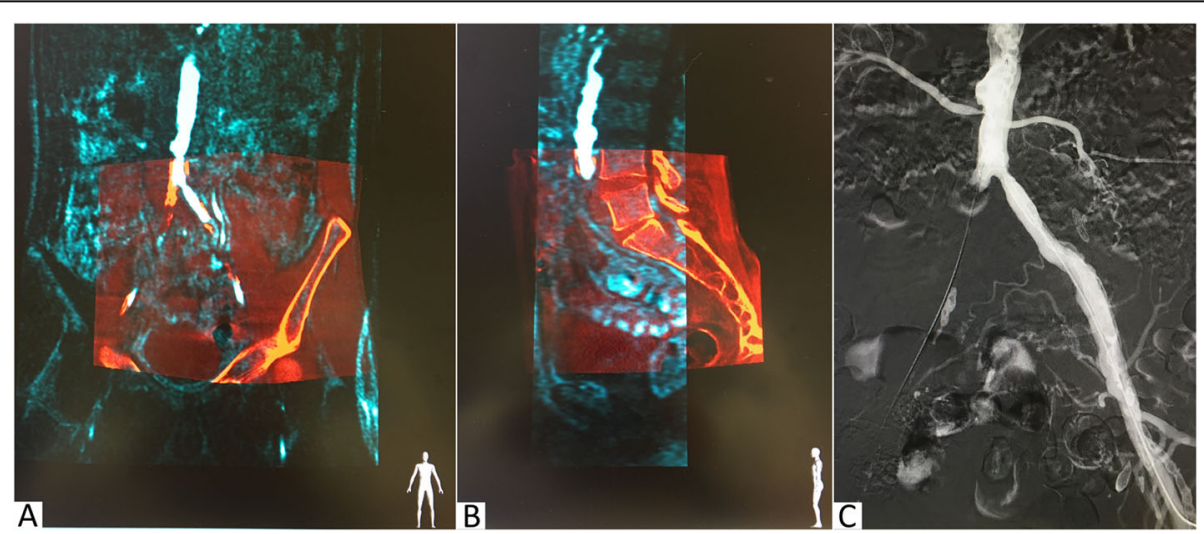

Fig. 1 Example of fluoroscopy with MRA fusion image guidance. $\mathbf{a}$, $\mathbf{b}$ Coronal and sagittal view of the image registration process (the cone-beam $\mathrm{CT}$ images in red and the MRA images in blue). c An example of the fusion image guidance during a digital subtraction angiography 


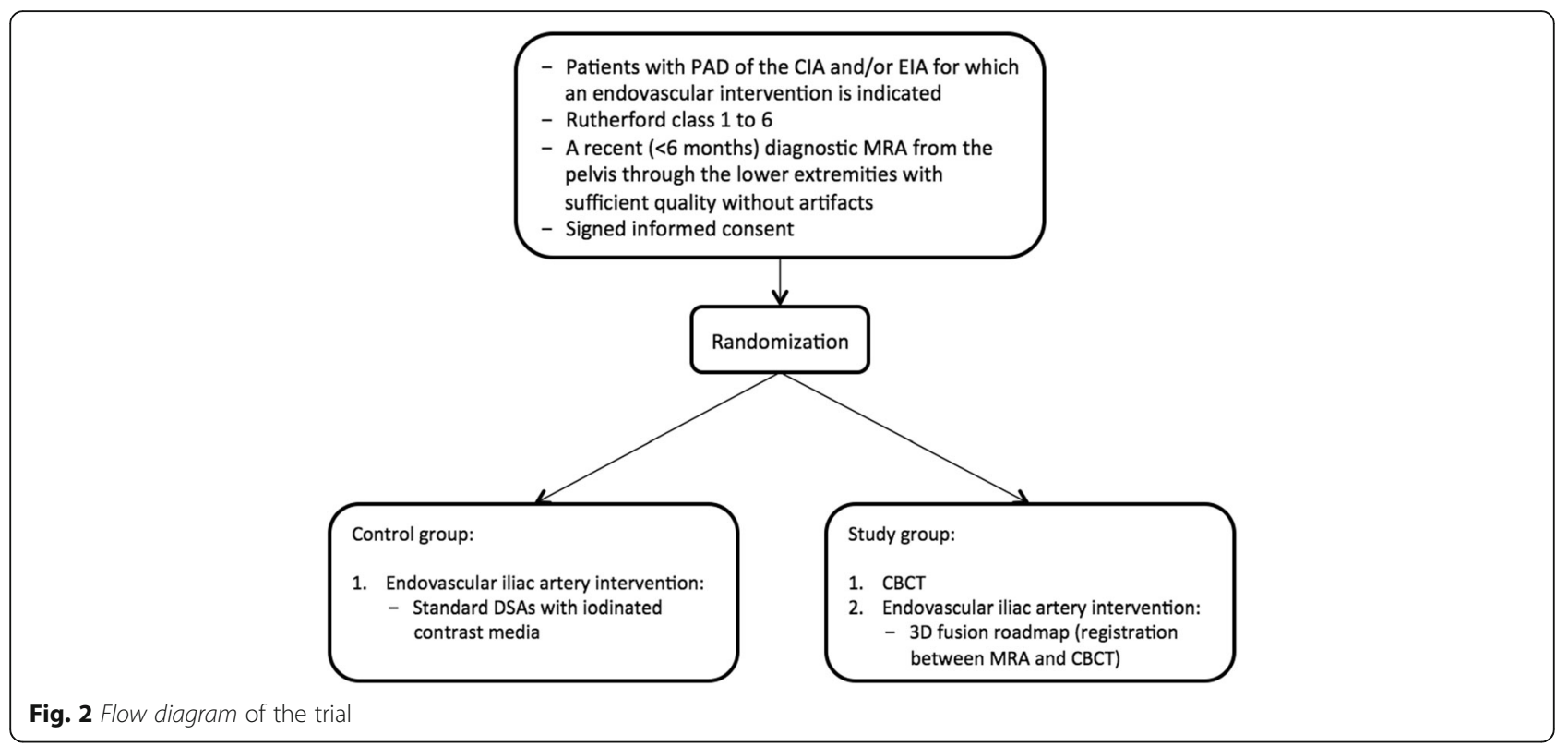

\section{Patients}

The study cohort will comprise 106 patients (> 18 years) with symptomatic common iliac artery (CIA) and/or external iliac artery (EIA) stenoses or occlusions and Rutherford category $1-6$, a recent ( $<6$ months) diagnostic MRA from the pelvis through the lower extremities, and an indication of endovascular intervention (PTA or recanalization). Participants will be randomized 1:1 to the control or study group (i.e. standard PTA or recanalization with or without the $3 \mathrm{D}$ image fusion technique). Patients will be included from the MUMC and SAH. Inclusion and exclusion criteria are summarized in Table 1.

\section{Randomization}

Randomization will be performed by the investigators with the use of an automated web-based randomization and electronic data capture software (MACRO 4.6.0, https://ecrf.ctcm.nl/macro). Randomization will be performed according to minimization; for a difference of 2 between the two groups (control and study group), there is a $90 \%$ chance that the next individual will be allocated to the group with the smallest number of patients. Stratification factors are the site (MUMC or SAH), weight ( $\leq 90$ or $>90 \mathrm{~kg}$ ), and estimated glomerular filtration rate $(\mathrm{eGFR})\left(\leq 60\right.$ or $\left.>60 \mathrm{~mL} / \mathrm{min} / 1.73 \mathrm{~m}^{2}\right)$. It is expected that the site, weight and eGFR have the biggest impact on the outcome measures. First, the groups should be similar regarding renal function because this may affect outcome. It can be argued that in patients with a reduced renal function, physicians are more careful with the amount of administered contrast. Thus, by using the eGFR as a stratification factor, this bias will be minimized. Second, the radiation dose is taken into account, which is dependent on the patients' weight. Therefore, stratification on weight is important. Third, it is not preferable to have one of the sites primarily performing the interventions with the $3 \mathrm{D}$ fusion technique and vice versa, since that would influence the user experience and is possibly also be related to the amount of contrast volume that is administered and the radiation dose that is used. Hence, these stratification factors were chosen to keep the distribution between the groups equal. Owing to the nature of the study, randomization will not be blinded for patients or physicians.

\section{Ethics}

The study will be conducted in accordance with the principles of the Declaration of Helsinki and the Medical Research Involving Human Subjects Act (in Dutch: Wet Medisch Wetenschappelijk Onderzoek met Mensen). The local medical ethical committees of both hospitals have approved the study protocol (NL47680.068.14, version 1.4). The study protocol was registered at the Netherlands Trial Register at www.trialregister.nl on 16 December 2014 (registration number: NTR5008). All patients will give written informed consent before study inclusion and randomization. Patient participation is voluntary; the participant can request to stop participation to the study at any time.

\section{Sample size calculation and statistical analysis}

The sample size was calculated using a two-tailed t-test with null hypothesis $E(D)=0$ (no difference between the groups) and alternative hypothesis $E(D) \neq 0$ (groups are 


\begin{tabular}{|c|c|c|c|c|}
\hline \multirow[b]{3}{*}{ TIMEPOINT** } & \multicolumn{4}{|c|}{ STUDY PERIOD } \\
\hline & \multirow{2}{*}{$\begin{array}{c}\text { Enrolment } \\
-1 \text { week }\end{array}$} & \multicolumn{3}{|c|}{ Day of the intervention } \\
\hline & & Pre & Peri & Post \\
\hline $\begin{array}{l}\text { ENROLMENT: } \\
\text { Eligibility screen } \\
\text { Informed consent } \\
\text { Allocation }\end{array}$ & $\begin{array}{l}x \\
x\end{array}$ & $X$ & & \\
\hline $\begin{array}{l}\text { INTERVENTIONS: } \\
\text { [Control group] } \\
\text { [Study group] }\end{array}$ & & & $\begin{array}{l}x \\
x\end{array}$ & \\
\hline $\begin{array}{r}\text { ASSESSMENTS: } \\
\text { agent } \\
\text { lodinated contrast } \\
\begin{array}{r}\text { Technical success } \\
\text { procedure }\end{array} \\
\text { Fluoroscopy time } \\
\text { Radiation dose } \\
\begin{array}{r}\text { Fusion accuracy } \\
\text { between overlaid } \\
\text { MRA and DSA } \\
\text { Complications } \\
\text { occurring during } \\
\text { the intervention } \\
\text { and before } \\
\text { discharge }\end{array}\end{array}$ & & & & $\begin{array}{l}X \\
X \\
x \\
x \\
x\end{array}$ \\
\hline $\begin{array}{r}\text { CLOSE OUT: } \\
\text { Hospital discharge }\end{array}$ & & & & $X$ \\
\hline
\end{tabular}

Fig. 3 Schedule of enrolment, interventions, and assessments*

Table 1 Eligibility criteria

\begin{tabular}{|c|c|}
\hline Inclusion & Exclusion \\
\hline $\begin{array}{l}\text { Patients with PAD of the CIA and/or EIA } \\
\text { for which an endovascular intervention is } \\
\text { indicated }\end{array}$ & Age $<18$ years \\
\hline Rutherford class 1-6 & $\begin{array}{l}\text { Treatment for acute arterial } \\
\text { ischemia }\end{array}$ \\
\hline $\begin{array}{l}\text { A recent ( }<6 \text { months) diagnostic MRA } \\
\text { from the pelvis through the lower } \\
\text { extremities with sufficient quality without } \\
\text { artifacts (e.g. in stent restenosis or patient } \\
\text { movement) }\end{array}$ & $\begin{array}{l}\text { Allergy for iodinated contrast } \\
\text { agents }\end{array}$ \\
\hline \multirow[t]{2}{*}{ Signed informed consent } & $\begin{array}{l}\text { Patients with aneurismal } \\
\text { lesions of the iliac artery }\end{array}$ \\
\hline & Pregnancy or breast-feeding \\
\hline
\end{tabular}

different). A type I error $(\alpha)$ of 0.05 with $\mathrm{z}$-value $Z_{\alpha} \approx$ 1.96 was accepted resulting in

$$
Z_{\alpha}=\sqrt{N} \frac{D}{\sigma_{D}}>1.96
$$

with $N$ the sample size and $\sigma_{D}$ the expected standard deviation. When assuming the alternative hypothesis is true $E(D)=\tau$ the power $(\pi(\tau))$ can be calculated by

$$
\pi(\tau)=1-\Phi\left(1.96-\sqrt{N} \frac{\tau}{\sigma_{D}}\right)
$$

with $\Phi$ being the Phi coefficient. When accepting a power of at least 0.90 , the alternative hypothesis for $E(D)$ $=\tau$ can be estimated by 


$$
\pi(\tau)=1-\Phi\left(1.96-\sqrt{N} \frac{\tau}{\sigma_{D}}\right)>0.90 .
$$

Alternatively, this can be written with as

$$
\Phi\left(1.96-\sqrt{N} \frac{\tau}{\sigma_{D}}\right)<0.10
$$

For a type II error of 0.1 , the z-value $\left(Z_{\beta}\right)$ becomes approximately 1.28

$$
\sqrt{N} \frac{\tau}{\sigma_{D}}>1.96+Z_{\beta}=1.96+1.28 \approx 3.24
$$

Given that half of the proportion of the study patients is randomized into the control group $\left(q_{0}=0.5\right)$ and the other half in the study group $\left(q_{1}=1-q_{0}=0.5\right)$, the sample size $(N)$ can then be estimated by

$$
N>\left(\frac{1}{q_{0}}+\frac{1}{q_{1}}\right)\left(\frac{3.24}{\tau} \sigma_{D}\right)^{2}
$$

Based on earlier research [9], we expect that $\tau=$ $20 \mathrm{~mL}$ of administered contrast volume may be reduced with the use of the 3D image fusion technique by reducing the number of contrast runs by at least two. Full-strength iodinated contrast is diluted 50:50 with a normal saline solution because a lower concentration of iodinated contrast agent has been shown not to compromise image quality during DSA of PAD patients [10]. To achieve a reduction of $20 \mathrm{~mL}$ per procedure with a mean volume of $60 \mathrm{~mL}$ and corresponding standard deviation of $\sigma_{D}=30 \mathrm{~mL}$, the sample size $N$ becomes a total of 96 patients. Therefore, 48 patients will be needed in each group. Adjusting for an anticipated loss to follow-up of $10 \%$, the total sample size will be 106 patients. An interim analysis will be performed halfway through, after inclusion of the first 40 patients, to assess the efficacy of the 3D fusion technique compared to the control group.

Normal distribution of the data will be tested. Normally distributed data will be represented as the mean and $95 \%$ confidence interval; non-normally distributed data will be represented as the median and interquartile range. Continuous variables will be analyzed with the independent samples $t$-test or Mann-Whitney $U$ test for normally and non-normally distributed data, respectively. Categorical data will be analyzed with the $\chi^{2}$ test. All tests will be two-sided and considered significant when $P<0.05$.

\section{Intervention}

The aim of the endovascular treatment is to obtain a patent CIA and/or EIA (i.e. $<30 \%$ residual stenosis or a reduction of the mean pressure gradient to $<10 \mathrm{mmHg}$ [11]), resulting in an improved blood flow to the legs. In case of a flow limiting dissection, $>30 \%$ residual stenosis, or acute elastic recoil after PTA, additional stent placement will be performed. If indicated, other arteries will be treated in addition to the CIA and/or EIA during the same intervention. The physician will choose an ipsilateral or contralateral technique (or rendezvous procedure), depending on the location of the lesion. The analysis will not include data of patients with solely diagnostic DSAs.

Before the start of the intervention, the patient will be placed supine on the angiography table. The contrast pump and table contrast agent will be diluted 50:50 with a normal saline solution. For patients randomized into the study group, a non-contrast-enhanced $\mathrm{CBCT}$ will be created at the beginning of the intervention using the flat panel detector of the $\mathrm{C}$-arm angiography system (Allura Xper FD20; Philips Healthcare). The CBCT will be automatically transferred to the 3D XtraVision workstation (Philips Healthcare). Subsequently, a manual 3D-3D registration will be performed between the preprocedural MRA and CBCT based on bony landmarks, such as vertebrae and the femoral heads, and the flow lumen on the MRA will be matched to the border of the calcifications as seen on the CBCT. The time required to perform the registration will be recorded.

After the registration, the physician will visually score the fusion accuracy of the entire iliac vasculature between the overlay and the initial DSA run. This fusion accuracy will be determined directly after the DSA is created and will be categorized as accurate $(<2 \mathrm{~mm})$, mismatch $(2-5 \mathrm{~mm})$, or inaccurate $(>5 \mathrm{~mm})$. An overlay that is inaccurate will be manually translated or reregistered to achieve a more accurate overlay that will be used during the intervention. Subsequently, the intervention will be performed according to the standard of care procedure and contrast runs will be performed when needed.

DSAs will be performed with $15 \mathrm{~mL}$ at $12 \mathrm{~mL} / \mathrm{s}$ or $8 \mathrm{~mL}$ at $4 \mathrm{~mL} / \mathrm{s}$ using a catheter in the aorta or CIA, respectively. A completion DSA will be created to confirm procedural success. DSA runs, whether or not in combination with the overlay (for the study group), will be stored. Primary and secondary endpoints will be registered, as well as baseline characteristics and procedural details, including ipsilateral or contralateral, length and location of the lesion, type of PTA balloon or stent, and number of stents.

All patients will receive 5000 IU of heparin during the intervention. The patients will receive bed rest after the intervention according to the physician's prescription. All patients will be prescribed acetylsalicylic acid (ASA or aspirin) at $80 \mathrm{mg} / \mathrm{d}$. The PTA and recanalization procedures are generally performed as outpatient procedures. Patient participation in the study will be completed after discharge. 
Any complication that occurs between the signed informed consent and hospital discharge will be recorded. There is no further follow-up period for this study.

\section{Data collection}

Data will be collected by use of an electronic case report form (eCRF), which will be prospectively maintained from the time the patient signs the informed consent until the completion of the study. Patient data will be anonymized; each individual will be given a unique study number (site code [MUMC/ANTO] and index number). Each center can only view and add eCRF data from its own site. At the end of the study, the eCRFs of both sites will be become available to both centers.

\section{Monitoring and adverse events}

A Data and Safety Monitoring Board (DSMB) will not be appointed for this study. A qualified monitor of the Clinical Trial Center Maastricht (CTCM) will monitor the study, which will ensure that the study is conducted according to ICH-GCP guidelines. Visits are conducted at the start of the study, after inclusion of five patients, halfway through, and at completion of the study. A monitoring report will be sent to the investigator after each visit.

Adverse events and serious adverse events will be recorded on the eCRFs. They will comprise a description of the event, type of complication (i.e. access site, systemic, organ-specific), severity, start date, end date, whether suspected to be intervention related, any action that is undertaken, and the outcome. Serious adverse events will be reported to the accredited METC through the "Toetsingsonline" page of the website of the Central Committee on Research Involving Human Subjects (in Dutch: Centrale Commissie Mensgebonden Onderzoek, CCMO [www.ccmo.nl]). The investigator will report serious adverse device events resulting from the use of the 3D image fusion technique to the accredited METC, the manufacturer, and the Inspection for Healthcare (in Dutch: Inspectie voor Gezondheidszorg [IGZ]).

\section{Discussion}

The use of angiography during endovascular interventions is essential to visualize stenotic or obstructive lesions [12]. With increasing complexity of procedures, the amount of iodinated contrast agent may increase. The potentially nephrotoxic effects of the iodinated contrast agent are especially problematic in patients with pre-existing renal failure $[2,13,14]$. A recent systematic review confirmed that the amount of administered iodinated contrast agent can be significantly reduced by using the 3D image technique during EVAR procedures [15]. In addition, the radiation dose, procedure time, and fluoroscopy time may be reduced during these procedures [15], which could be beneficial to physicians, who receive high exposure to radiation throughout their practice.

A vacuum mattress can be used to reduce patient movement [16], which can minimize overlay inaccuracy caused by movement artifacts. We assume that movement artifacts will be smaller in the pelvic area than in the legs and that registration in this area will be less challenging because of the bony structures (vertebrae, femoral head, and iliac crest) and larger diameters of the arteries. This research focuses on the iliac arteries; when this study demonstrates the benefits of the 3D fusion technique in endovascular iliac artery interventions, the technique can be extended to femoro-crural lesions.

An alternative to iodinated contrast agents is the use of angiography based on carbon dioxide $\left(\mathrm{CO}_{2}\right)$. The use of $\mathrm{CO}_{2}$ during EVAR procedures is safe and reliable and may be especially beneficial for patients with renal insufficiency [17]. However, variability in image quality has been reported and a careful technique is required for each injection to prevent air contamination. Additionally, there is a possibility of pain after injection, and $\mathrm{CO}_{2}$ for intravascular use has the potential for air embolism, which could result in ischemia or tissue infarction [18]. Although $\mathrm{CO}_{2}$ may be able to replace nephrotoxic contrast agents and reduce iodine contrast agent costs $[18,19]$, it will probably not reduce the radiation doses to patient and staff. Carbon dioxide and the 3D image fusion technique can be complementary during (complex) EVAR [20, 21]; however, 3D fusion in combination with $\mathrm{CO}_{2}$ for iliac artery interventions has not yet been investigated.

Preoperative MRA or CTA can be used for 3D image guidance. Registration between two similar image modalities (i.e. CTA to CBCT) may be less challenging than registration between MRA and CBCT, because MRA visualizes the flow lumen of the arteries, instead of calcified speculae. Nevertheless, because an MRA of the lower abdomen, pelvis, and lower extremities is the standard diagnostic imaging in the preoperative workflow of many patients with PAD, only patients with preoperative MRA datasets will be included; therefore, patients with contraindications to MRA are not eligible. Furthermore, because of susceptibility artifacts on the MRA, in-stent stenoses cannot be judged and those patients will not be included in this trial. No additional preoperative imaging needs to be performed for this study and patient burden is minimized.

Image registration can be performed to a CBCT (3D-3D image fusion) or two fluoroscopic orthogonal projections (2D-3D image fusion). The acquisition of a CBCT and registration process takes approximately $5-10 \mathrm{~min}$ and is reported to be more accurate than 2D-3D image fusion [20]. However, the latter is reported to be fast and can be performed with a minimal effective dose of $0.14-0.20 \mathrm{mSv}$ [21]. Sailer et al. [22] reported that a CBCT corresponds 
to a mean effective dose of $3.5 \mathrm{mSv}$ (95\% confidence interval, 3.0-4.1 mSv), whereas van den Berg [21] reported an effective dose of $1.53-1.66 \mathrm{mSv}$. The patient's radiation dose from a CBCT may add $21 \%$ of the total procedural DAP for iliac artery interventions [9], which is comparable to approximately $7 \mathrm{~min}$ of fluoroscopy time [22]. During fluoroscopy, the physician is at the tableside, but is usually far away from the $\mathrm{C}$-arm during the acquisition of the $\mathrm{CBCT}$. When the $3 \mathrm{D}$ fusion technique is used during EVAR, the fluoroscopy time could be significantly reduced $[23,24]$. This reduction may also be feasible for iliac artery interventions, which will be beneficial to both the operator and the patient, and could potentially result in a reduction of costs. The possible benefit for contrast saving, combined with the possible reduction of fluoroscopy time might outweigh the additional radiation dose of a CBCT.

\section{Trial status}

The METC has approved the study protocol. Currently, 40 patients have been enrolled in the 3DMR-Iliac-Roadmapping study. The expected project completion for this trial is December 2018.

\section{Additional file}

Additional file 1: SPIRIT 2013 Checklist: Recommended items to address in a clinical trial protocol and related documents*. (DOC $121 \mathrm{~kb}$ )

\section{Abbreviations}

2D: Two-dimensional; 3D: Three-dimensional; ASA: Acetylsalicylic acid; CBCT: Cone-beam computed tomography; CCMO: Centrale Commissie Mensgebonden Onderzoek; $\mathrm{CIA}$ : Common iliac artery; $\mathrm{CO}_{2}$ : Carbon dioxide; CTA: Computed tomography angiography; CTCM: Clinical Trial Center Maastricht; DAP: Dose area product; DCMB: Data and Safety Monitoring Board; DSA: Digital subtraction angiography; eCRF: Electronic case report form; EIA: External iliac artery; EVAR: Endovascular abdominal aortic aneurysm repair; IGZ: Inspectie voor Gezondheidszorg; MRA: Magnetic resonance angiography; MUMC: Maastricht University Medical Center; PAD: Peripheral artery disease; PTA: Percutaneous transluminal angiography; SAH: St. Antonius Hospital

\section{Acknowledgements}

Not applicable.

\section{Funding}

The trial will be self-funded by the investigators.

\section{Availability of data and materials}

Not applicable.

\section{Authors' contributions}

$\mathrm{SH}, \mathrm{MH}, \mathrm{AS}, \mathrm{DH}, \mathrm{MS}$, and J-PV developed the protocol for the study. SG, SH, and $\mathrm{MH}$ are involved in data collection. SG and $\mathrm{SH}$ drafted the manuscript. $\mathrm{MH}, \mathrm{AS}, \mathrm{DH}, \mathrm{MS}$, and J-PV critically reviewed and revised the draft report. All authors read and approved the final manuscript.

\section{Ethics approval and consent to participate}

The study will be conducted in accordance with the principles of the Declaration of Helsinki and the Medical Research Involving Human Subjects Act (in Dutch: Wet Medisch Wetenschappelijk Onderzoek met Mensen). The local medical ethical committees of both hospitals have approved the study protocol (NL47680.068.14, version 1.4). All patients will give written informed consent before study inclusion and randomization.

Consent for publication

Not applicable.

Competing interests

$\mathrm{MH}$ reports an institutional research grant from Philips Healthcare.

\section{Publisher's Note}

Springer Nature remains neutral with regard to jurisdictional claims in published maps and institutional affiliations.

\section{Author details}

${ }^{1}$ Department of Vascular Surgery, St. Antonius Hospital, Koekoekslaan 1, 3435 CM Nieuwegein, The Netherlands. ${ }^{2}$ Department of Radiology, Maastricht University Medical Center, Maastricht, The Netherlands. ${ }^{3}$ Department of Radiology, Stanford University School of Medicine, Stanford, CA, USA. ${ }^{4}$ Department of Radiology, St. Antonius Hospital, Nieuwegein, The Netherlands. ${ }^{5}$ Department of Surgery, Division of Vascular Surgery, University Medical Center Groningen, Groningen, The Netherlands.

Received: 5 December 2017 Accepted: 13 October 2018

Published online: 01 November 2018

References

1. Bekken J, Jongsma H, Ayez N, Hoogewerf C, Van Weel V, Fioole B. Angioplasty versus stenting for iliac artery lesions (Review). Cochrane Database Syst Rev. 2015;5:1-29 https://doi.org/10.1002/14651858.CD007561. pub2.www.cochranelibrary.com.

2. Solomon R, Dumouchel W. Contrast media and nephropathy: findings from systematic analysis and Food and Drug Administration reports of adverse effects. Investig Radiol. 2006;41(8):651-60 https://doi.org/10.1097/01.rli. $0000229742.54589 .7 \mathrm{~b}$.

3. Soulez G, Therasse E, Giroux M-F, Bouchard L, Gilbert P, Perreault P, et al. Management of peripheral arterial disease: Role of computed tomography angiography and magnetic resonance angiography. Presse Med. 2011;40(9): e437-52 https://doi.org/10.1016/j.lpm.2010.10.037.

4. Kobeiter $\mathrm{H}$, Nahum J, Becquemin J-P. Zero-contrast thoracic endovascular aortic repair using image fusion. Circulation. 2011;124:e280-2.

5. Dijkstra ML, Eagleton MJ, Greenberg RK, Mastracci T, Hernandez A. Intraoperative C-arm cone-beam computed tomography in fenestrated/ branched aortic endografting. J Vasc Surg. 2011;53:583-90 https://doi.org/ 10.1016/j.jvs.2010.09.039.

6. Bargellini I, Turini F, Bozzi E, Lauretti D, Cicorelli A, Lunardi A, et al. Image fusion of preprocedural CTA with real-time fluoroscopy to guide proper hepatic artery catheterization during transarterial chemoembolization of hepatocellular carcinoma: A feasibility study. Cardiovasc Intervent Radiol. 2013;36:526-30 https://doi.org/10.1007/s00270-012-0495-9.

7. Sadek M, Berland TL, Maldonado TS, Rockman CB, Mussa FF, Adelman MA et al. Use of preoperative magnetic resonance angiography and the Artis zeego fusion program to minimize contrast during endovascular repair of an iliac artery aneurysm. Ann Vasc Surg. 2014;28:261.e1-5 https://doi.org/10. 1016/j.avsg.2013.07.001.

8. Sailer AM, De Haan MW, Peppelenbosch AG, Jacobs MJ, Wildberger JE, Schurink GWH. CTA with fluoroscopy image fusion guidance in endovascular complex aortic aneurysm repair. Eur J Vasc Endovasc Surg. 2014:47:349-56 https://doi.org/10.1016/j.ejvs.2013.12.022.

9. Sailer AM, de Haan MW, de Graaf R, van Zwam WH, Schurink GWH, Nelemans PJ, et al. Fusion guidance in endovascular peripheral artery interventions: a feasibility study. Cardiovasc Intervent Radiol. 2014;38:314-21 https://doi.org/10.1007/s00270-014-0951-9.

10. Jens S, Schreuder SM, De Boo DW, van Dijk LC, van Overhagen H, Bipat S, et al. Lowering iodinated contrast concentration in infrainguinal endovascular interventions: a three-armed randomized controlled non-inferiority trial. Eur Radiol. 2016;26(8):2446-54 https://doi.org/10.1007/s00330-015-4109-1.

11. Stoner MC, Calligaro KD, Chaer RA, Dietzek AM, Farber A, Guzman RJ, et al. Reporting standards of the Society for Vascular Surgery for endovascular treatment of chronic lower extremity peripheral artery disease. J Vasc Surg. 2016;64(1):e1-e21 https://doi.org/10.1016/j.jvs.2016.03.420. 
12. Törnqvist P, Dias NV. TR. Optimizing imaging for aortic repair. J Cardiovasc Surg. 2015;56:189-95.

13. Gleeson TG, Bulugahapitiya S. Contrast-induced nephropathy. Am J Roentgenol. 2004;183(December):1673-89 https://doi.org/10.1016/B978-14377-1367-1.00117-8.

14. Laskey WK, Jenkins C, Selzer F, Marroquin OC, Wilensky RL, Glaser R, et al. Volume-to-creatinine clearance ratio. A Pharmacokinetically Based Risk Factor for Prediction of Early Creatinine Increase After Percutaneous Coronary Intervention. J Am Coll Cardiol. 2007;50(7):584-90 https://doi.org/ 10.1016/j.jacc.2007.03.058.

15. Goudeketting SR, Heinen SGH, van den Heuvel DAF, de Vries JPPM, van Strijen MJ, Sailer AM. Pros and cons of 3D image fusion in endovascular aortic repair: a systematic review and meta-analysis. J Endovasc Ther. 2017; 24:595-603 https://doi.org/10.1177/1526602817708196.

16. Mallman C, Wolf K-J, Wacker FK, Meyer BC. Assessment of patient movement in interventional procedures using electromagnetic detection comparison between conventional fixation and vacuum mattress [in German]. Fortschr Röntgenstr. 2012;184:37-41.

17. Criado E, Upchurch GR, Young K, Rectenwald JE, Coleman DM, Eliason JL, et al. Endovascular aortic aneurysm repair with carbon dioxide-guided angiography in patients with renal insufficiency. J Vasc Surg. 2012;55(6): 1570-5 https://doi.org/10.1016/j.jvs.2011.11.142.

18. Ahmad W, Gawenda M, Brunkwall S, Shahverdyan R, Brunkwall JS. Endovascular aorto-liac aneurysm repair with fenestrated stent graft and iliac side branch using image fusion without iodinated contrast medium. Ann Vasc Surg. 2016:33:231.e5-9 https://doi.org/10.1016/j.avsg.2015.11.031.

19. Koutouzi G, Hendrikson O, Roos H, Zachrisson K, Falkenberg M. EVAR guided by 3D image fusion and CO2 DSA, a new imaging combination for patients with renal insuffiency. J Endovasc Ther. 2015;22:912-7.

20. Schulz CJ, Schmitt M, Böckler D, Geisbüsch P. Feasibility and accuracy of fusion imaging during thoracic endovascular aortic repair. J Vasc Surg. 2015 63:314-22 https://doi.org/10.1016/j.jvs.2015.08.089.

21. van den Berg JC. Update on new tools for three-dimensional navigation in endovascular procedures. Aorta. 2014;2:279-85 https://doi.org/10.12945/j. aorta.2014.14-054.

22. Sailer AM, Schurink GWH, Wildberger JE, de Graaf R, van Zwam WH, de HAan MW, et al. Radiation exposure of abdominal cone beam computed tomography. Cardiovasc Intervent Radiol. 2014;38(1):112-20 https://doi.org/ 10.1007/s00270-014-0900-7.

23. McNally MM, Scali ST, Feezor RJ, Neal D, Huber TS, Beck AW. Threedimensional fusion computed tomography decreases radiation exposure, procedure time, and contrast use during fenestrated endovascular aortic repair. J Vasc Surg. 2015;61:309-16 https://doi.org/10.1016/j.jvs.2014.07.097.

24. Stangenberg L, Shuja F, Carelsen B, Elenbaas T, Wyers MC, Schermerhorn ML. A novel tool for three-dimensional roadmapping reduces radiation exposure and contrast agent dose in complex endovascular interventions. $J$ Vasc Surg. 2015;62:448-55 https://doi.org/10.1016/j.jvs.2015.03.041.

Ready to submit your research? Choose BMC and benefit from:

- fast, convenient online submission

- thorough peer review by experienced researchers in your field

- rapid publication on acceptance

- support for research data, including large and complex data types

- gold Open Access which fosters wider collaboration and increased citations

- maximum visibility for your research: over $100 \mathrm{M}$ website views per year

At $\mathrm{BMC}$, research is always in progress.

Learn more biomedcentral.com/submissions 